\title{
ORIGINAL
}

\section{GANANCIA FUNCIONAL Y ESTANCIA HOSPITALARIA EN LA UNIDAD GERIATRICA DE MEDIA ESTANCIA DEL HOSPITAL CENTRAL DE CRUZ ROJA DE MADRID}

Juan J. Baztán, Juan R. Domenech, Margarita González, Silvia Forcano, Carmen Morales, Isidoro Ruipérez

Servicio de Geriatría. Hospital Central Cruz Roja. Madrid.

\section{RESUMEN}

Fundamento: Las unidades geriátricas de media estancia o convalecencia fueron definidas por el Insalud en 1996 como aquel nivel asistencial geriátrico hospitalario destinado a restablecer aquella funciones, actividades o secuelas, alteradas como resultado de diferentes procesos previos. El objetivo de este trabajo es evaluar las características de los pacientes asociadas a la ganancia funcional y estancia en las unidades geriátricas de media estancia.

Métodos: Se estudió a todos los pacientes ingresados entre mayo de 2000 y diciembre de 2001. Se evaluó la ganancia funcional semanal y global con el Indice de Barthel, la estancia hospitalaria y la eficiencia (IB al alta-IB al ingreso/estancia). Se estableció como umbral de eficacia la mejora en la ganancia semanal > 5 puntos en IB.

Resultados: Fueron evaluados 459 pacientes con una edad media de edad de 80,56 $( \pm 7,45)$ ingresados para recuperación funcional de secuelas de ictus $(48,4 \%)$, patología ortopédica $(26,3 \%)$ e inmovilismo por otras patologías $(23,5 \%)$. La ganancia funcional total fue de $29,71( \pm 16,75)$ puntos en el Indice de Barthel, con una estancia media de $24,93( \pm 12,94)$ días y una eficiencia de 1,44 $( \pm 1,02)$. La ganancia funcional semanal estuvo por encima de umbral establecido en las tres primeras semanas, independientemente de la edad y patología motivo de ingreso. En el análisis de regresión multivariante la edad, el ingreso por ictus, el deterioro funcional previo y cognitivo al ingreso, la comorbilidad y demora en el ingreso se asociaron a menor ganancia funcional. El ingreso por ictus y la mejor situación funcional previa y cognitiva al ingreso se asociaron a mayor estancia.

Conclusiones: La estancia hospitalaria en unidades geriátricas de media estancia es adecuada, al menos, en las tres primeras semanas. La comparación de los resultados entre unidades debiera ajustarse por edad, patología motivo de ingreso, comorbilidad y situación funcional y cognitiva de los pacientes.

Palabras clave: Servicios de salud para los ancianos, rehabilitación, hospital, eficiencia, anciano frágil.

Correspondencia:

Dr. Juan J. Baztán

S Geriatría. Hospital Central Cruz Roja.

Avda. Reina Victoria 26

28003 - MADRID

Correo electrónico: baztanhornillos@eresmas.net
ABSTRACT

Functional Gain and Length of Hospital Stay at a Medium-Stay Geriatric Care Unit at the Central Red Cross Hospital in Madrid, Spain

Background: The medium-stay or convalescent care geriatric units were defined by the Spanish National Health Institute in 1996 as being the level of geriatric hospital care aimed at recovering those functions, activities or sequelae having undergone changes as a result of different prior processes. This study is aimed at evaluating the characteristics of patients related to functional gain and stay in medium-stay geriatric units.

Methods: A study was made of all those patients admitted throughout the May 2000-December 2001 period. The weekly and overall functional gain was evaluated using the Barthel Index (BI), the hospital stay and the effectiveness (BI at discharge-BI at admisthe hospital stay and the effectiveness (BI at discharge-BI at admis-
sion/during stay) having been evaluated. An improvement in the weekly gain of $\mathrm{BI}>5$ points was set at the effectiveness threshold.

Results: A total of 459 patients averaging age $80.56( \pm 7.45)$ admitted for functional recovery from sequelae of ictus $(48.4 \%)$, orthopedic disorders $(26,3 \%)$ and immobility due to other ailments $(23.5 \%)$ were evaluated. The total functional gain was 29.71 $( \pm 16.75)$ Barthel Index points, entailing an average stay of 24.93 $( \pm 12.94)$ days and a $1.44( \pm 1.02)$ effectiveness. The weekly functional gain was above the threshold set during the first three weeks, independently of the age and disorder for which admitted. In the multivariate regression analysis, the age, admission due to ictus, functional impairment prior to admission, cognitive impairment a admission, comorbility and delay in admission were related to a lesser functional gain. Admission due to ictus and a better functional condition prior to admission and better cognitive condition at admission were related to a longer stay.

Conclusions: Hospital stays in medium-stay geriatric units is adequate, at least during the first three weeks. A comparison of the results among units should be adjusted by age, the disorder for which admitted, comorbility and functional and cognitive condition of the patients.

Key words: Heath services for aged. Rehabilitation outcome. Hospital. Efficiency. Frail elderly. 


\section{INTRODUCCIÓN}

Las unidades geriátricas de media estancia o convalecencia fueron definidas por el Insalud en 1996 como aquel nivel asistencial geriátrico hospitalario destinado a restablecer aquellas funciones, actividades o secuelas, alteradas como resultado de diferentes procesos previos (médicos, quirúrgicos o traumatológicos) ${ }^{1}$. Dado que la terminología de «media estancia o convalecencia» es específica de nuestro ámbito sanitario, el diseño y funcionamiento de estas unidades geriátricas correspondería al de otras unidades descritas en la literatura como de rehabilitación geriátrica, cuidados posagudos, de transición o interme$\operatorname{dios}^{2}$.

Si bien los objetivos primordiales de estas unidades son la recuperación funcional y la reducción de la incidencia de institucionalización definitiva, la consecución de estos objetivos deben ajustarse a un tiempo de atención no muy prolongado, con objeto de aumentar la eficiencia de estas unidades. La mejora en la eficiencia no es un objetivo menor, dada la dificultad en la implantación de estas unidades hospitalarias, derivada en gran medida del desplazamiento de los recursos hospitalarios hacia cuidados agudos $^{3,5}$. En este sentido, la evaluación de factores clínicos, funcionales, mentales y sociales es uno de los aspectos básicos para optimizar el rendimiento de estas unidades ${ }^{3,6}$.

La adecuación de la estancia hospitalaria en unidades de agudos ha sido ampliamente estudiada en la literatura mediante la aplicación de instrumentos como la Appropriateness Evaluation Protocol, que consideran únicamente razones médicas relacionadas con la hospitalización por cuidados agudos, sin considerar otros factores funcionales, mentales o sociales ${ }^{7}$. Sin embargo, estos últimos factores adquieren especial importancia a la hora de evaluar la justificación de la estancia en otras unidades hospitalarias dedicadas a cuidados no agudos ${ }^{8}$. Así, en unidades geriátricas de media estancia donde el principal objetivo asistencial es la recuperación del deterioro funcional, es necesario desarrollar instrumentos para la evaluación de la estancia hospitalaria que contemplen factores diferentes a los empleados para las unidades de hospitalización aguda. Algunos autores han postulado que la aplicación del índice de Barthel podría ser un instrumento de evaluación eficaz en estas unidades, considerando que el ingreso sería adecuado mientras los pacientes consiguieran una mejoría de su situación funcional (ganancia funcional) mayor de 5 puntos a la semana $^{9,10}$.

La eficiencia de la ganancia funcional, entendida como la relación entre la ganancia funcional obtenida y el tiempo necesario para lograrla (estancia hospitalaria), es un parámetro con frecuencia utilizado en la evaluación de estas unidades ${ }^{11,16}$. Este indicador es complementario a los indicadores de efectividad (ganancia funcional e incidencia de institucionalización al alta principalmente), pero puede servir como medida objetiva en la comparación de utilización de recursos entre diferentes unidades. Sin embargo, tanto la ganancia funcional como la estancia hospitalaria y la eficiencia pueden estar condicionadas por la complejidad de las características de los pacientes $y$, por tanto, para ajustar su interpretación es necesario conocer su relación con características clínicas, funcionales, mentales y sociales de los pacientes atendi$\operatorname{dos}^{13}$.

El objetivo de nuestro estudio es evaluar la estancia hospitalaria mediante la descripción de la evolución funcional semanal de los pacientes atendidos en una unidad geriátrica de media estancia hospitalaria. De forma añadida nos planteamos conocer las características de los pacientes al ingreso asociadas con la ganancia funcional, estancia hospitalaria y eficiencia de la ganancia funcional. 


\section{SUJETOS Y MÉTODOS}

\section{Pacientes}

Se estudió a todos los pacientes ingresados consecutivamente en la Unidad de Media Estancia del Servicio de Geriatría del Hospital Central de Cruz Roja de Madrid desde mayo de 2000 hasta diciembre de 2001. Esta unidad dispone de 30 camas y está adaptada para la atención de ancianos frágiles con incapacidad. El médico responsable de la unidad es un especialista en geriatría y está atendida por personal de enfermería entrenado en el manejo de problemas geriátricos, 2 terapeutas ocupacionales y 1 trabajadora social a tiempo parcial, que trabajan en colaboración estrecha con el servicio de rehabilitación del hospital (médico rehabilitador, fisioterapeutas y logoterapeutas). Todos ellos trabajan de forma interdisciplinaria, valorando a los pacientes previamente al ingreso y posteriormente en las 48 primeras horas de la estancia en la unidad, con sesiones semanales para monitorizar la evolución de los pacientes y planificar precozmente los cuidados al alta.

Los criterios de ingreso en esta unidad fueron ser pacientes geriátricos con deterioro funcional moderado-severo potencialmente reversible, estables clínicamente y con ausencia de deterioro cognitivo previo severo o enfermedad médica en situación terminal.

\section{Variables de estudio}

Al ingreso se recogieron datos sociodemográficos, clínicos, funcionales y sociales. La patología principal causante de la incapacidad que motivó el ingreso se categorizó como ictus, patología ortopédica (fracturas de cadera y otras), e inmovilidad secundaria a otros procesos médicos o quirúrgicos. La comorbilidad fue evaluada con el índice de Charlson ${ }^{17}$. La situación funcional previa al inicio de la patología incapaci- tante, al ingreso y al alta fue evaluada mediante el índice de Barthel (IB) en su versión modificada por $\mathrm{Shah}^{18}$, que evalúa 10 actividades básicas de la vida diaria con una puntuación que oscila entre 0 (máxima dependencia) y 100 (máxima independencia) ${ }^{19}$. La ganancia funcional al alta fue calculada como la diferencia entre el índice de Barthel al alta respecto al del ingreso. Se recogió igualmente el tiempo transcurrido desde el inicio de la patología causante de la incapacidad y el ingreso en la unidad. El estado mental al ingreso fue evaluado mediante el test de Pfeiffer, que evalúa la función cognitiva con un punto de corte de 5 ó más para la presencia de deterioro moderado-severo 20,21 . La situación social fue evaluada a través de la escala sociofamiliar de Gijón modificada ${ }^{22}$, que evalúa la situación familiar, vivienda, relaciones y contactos sociales, apoyos de la red social y situación económica, con una puntuación que oscila entre 0 y 20 (mayor precariedad social).

\section{Variables de resultado:}

Se evaluó la ganancia funcional semanal determinada por la diferencia entre la puntuación del índice de Barthel de cada evaluación en relación a la realizada la semana previa. Se estableció como estándar de eficacia una ganancia funcional semanal mayor de 5 puntos en el índice de Barthel, como propusieron Granger y Hamilton ${ }^{9}$.

Dado que el intervalo entre la primera medida y el ingreso podía oscilar entre 4 y 9 días y que la posterior evaluación semanal podía realizarse entre 6-8 días después de la previa, se estableció como parámetro de ajuste temporal la ganancia funcional medida por días de estancia. Este parámetro se denomina en la literatura referida a la actividad de unidades hospitalarias con orientación rehabilitadora como «eficiencia de la ganancia funcional» $\mathrm{y}$ viene definida como el cociente entre la ganancia funcional y los días de estancia ${ }^{11-16}$. La «eficiencia de la 
ganancia funcional» fue calculada semanalmente, asumiendo como estándar de eficiencia el valor mayor de 0,7 porque aproximadamente coincide con el estándar de ganancia funcional diaria referido anteriormente.

\section{Análisis estadístico:}

Para la descripción general de la muestra las variables cuantitativas se expresaron en mediana y rango intercuartílico. La ganancia funcional y eficiencia semanal se expresaron en medias y error estándar, utilizando la «t» de Student y test de ANOVA para su comparación, en función del número de categorías de las variables basales, considerando la existencia de significación estadística si p<0,05.

Por último, se realizó un análisis multivariante de regresión lineal, incluyendo todas las variables basales (previas y al ingreso) consideradas, para evaluar su relación con la ganancia funcional global, estancia hospitalaria y eficiencia de la ganancia funcional.

Los datos fueron analizados en el paquete estadístico SPSS.9.

\section{RESULTADOS}

Durante el periodo de estudio ingresaron 506 pacientes en la unidad, de los cuales se excluyeron 47 para el análisis (31 derivados al alta a unidades de agudos, 7 permanecieron menos de 5 días en la unidad, 5 fallecieron y 4 fueron excluidos por datos insuficientes). Las características de los 459 pacientes que constituyen la población final del estudio se presentan en la tabla 1. La edad media fue 80,56 años $(7,45)$, con una ganancia funcional total de $29,71(16,75)$ puntos en el Indice de Barthel, una estancia media de $24,93(12,94)$ días y una eficiencia media de $1,44(1,02)$.

En la figura 1 se muestra la evolución semanal de la ganancia funcional, destacando que en las tres primeras semanas está por encima del umbral de eficacia considerado (ganancia de $>5$ puntos en el índice de Barthel). Esta ganancia funcional semanal ajustada por tiempo de estancia (lo que denominamos eficiencia de la ganancia funcional) se presenta en la figura 2 , mostrando un perfil similar a la anterior. La presencia del pico de ganancia funcional que se produce en la octava semana corresponde a una población marginal de la muestra (por encima del percentil 90 de la estancia).

En las figuras 3 y 4 se presenta el perfil de evolución semanal de la muestra categorizada por tramos de edad (figura 3 ) y patología principal motivo de ingreso (figura 4). En relación con la edad, destaca como el perfil de la ganancia funcional semanal es mayor y más mantenido en el grupo de menor edad, aunque sin diferencias significativas entre grupos en ninguno de los puntos de corte semanales. Pese a ello, incluso el grupo de los muy ancianos (mayores de 84 años) se mantiene por encima del umbral de eficacia de la ganancia funcional semanal en las tres primeras semanas de ingreso.

Los pacientes con patología ortopédica obtienen una ganancia funcional mayor en las primeras semanas, pero ésta sigue un descenso progresivo frente a los pacientes con ictus o inmovilidad por otras causas, patologías en las que los pacientes que permanecen en la unidad presentan una mejoría mantenida en las semanas posteriores. No hubo diferencias estadísticamente significativas en los puntos de corte de ganancia funcional semanal entre grupos, salvo en la tercera semana entre los pacientes con ictus y aquellos con inmovilismo $(\mathrm{p}<0,05)$.

Por último se analizó la evolución de la ganancia funcional semanal de los pacientes de la muestra procedentes de otras unidades hospitalarias $(81,9 \%)$ en función de la unidad de procedencia (figura 5). Las personas remitidas de la unidad geriátrica de agudos $(n=106)$ tuvieron una ganancia funcional en la primera semana significativamente menor 
Tabla 1

Características generales de los pacientes

\begin{tabular}{|c|c|}
\hline $\mathrm{N}$ & 459 \\
\hline Edad & $80(75-86)$ \\
\hline Mujeres & $64,9 \%$ \\
\hline Indice Barthel Previo & $95(84-100)$ \\
\hline Indice de Barthel Ingreso & $28(17-44)$ \\
\hline Indice de Barthel al Alta & $63(43-78)$ \\
\hline SPMSQ Pfeiffer al ingreso & $2(1-5)$ \\
\hline Procedencia hospitalaria & $81,9 \%$ \\
\hline $\begin{array}{l}\text { Motivo de Ingreso: } \\
-\quad \text { Ictus } \\
-\quad \text { Patología ortopédica } \\
-\quad \text { Inmovilidad por otras causas }\end{array}$ & $\begin{array}{l}48,4 \% \\
26,3 \% \\
23,5 \%\end{array}$ \\
\hline $\begin{array}{l}\text { Estancia (días): } \\
\text { - } \quad \text { Edad: } \\
<75 \text { años } \\
75-84 \text { años } \\
\geq 85 \text { años } \\
\text { Motivo de ingreso: } \\
\text { ictus } \\
\text { patología ortopédica } \\
\text { inmovilidad por otras causas }\end{array}$ & $\begin{array}{c}22(15-33) \\
19,5(14-35,75) \\
24(15,75-34) \\
21(15-30) \\
27(17-38) \\
21(14-30,5) \\
18(13-26) \\
\end{array}$ \\
\hline Ganancia Funcional Total & $29(18-39)$ \\
\hline $\begin{array}{l}\text { Eficiencia de la ganancia funcional } \\
\text { - } \quad \text { Edad: } \\
<75 \text { años } \\
75-84 \text { años } \\
\geq 85 \text { años } \\
\text { - Motivo de ingreso: } \\
\text { ictus } \\
\text { patología ortopédica } \\
\text { inmovilidad por otras causas }\end{array}$ & $\begin{array}{c}1,29(0,71-2) \\
1,39(0,83-2,24) \\
1,31(0,74-2,01) \\
1,08(0,65-1,63) \\
1(0,61-1,63) \\
1,55(1,05-2,24) \\
1,34(0,77-2,04)\end{array}$ \\
\hline
\end{tabular}

Datos cuantitativos presentados como mediana (rango intercuartílico)

$(\mathrm{p}<0,05)$ que los procedentes de otras unidades hospitalarias $(n=262)$.

Finalmente, los resultados de los análisis multivariantes entre variables basales y ganancia funcional global, estancia y eficiencia de la ganancia se presentan en la tabla 2. Una peor situación funcional previa y mental al ingreso junto con mayor comorbilidad y demora del ingreso se asociaron con menor ganancia funcional al alta. El ingreso por ictus y la mejor situación funcio- nal previa a la patología incapacitante se asociaron a una mayor estancia y, por el contrario, una mejor situación funcional y peor mental al ingreso se relacionaron con menor estancia en la unidad. Por último, el ingreso por ictus y la demora en el ingreso en la unidad se asociaron a una menor eficiencia. La edad no se asoció de forma significativa con ninguno de los tres parámetros de resultado, aunque había una tendencia entre el aumento de edad y una menor ganancia funcional y eficiencia de la misma al alta. 
Figura 1

Características generales de los pacientes

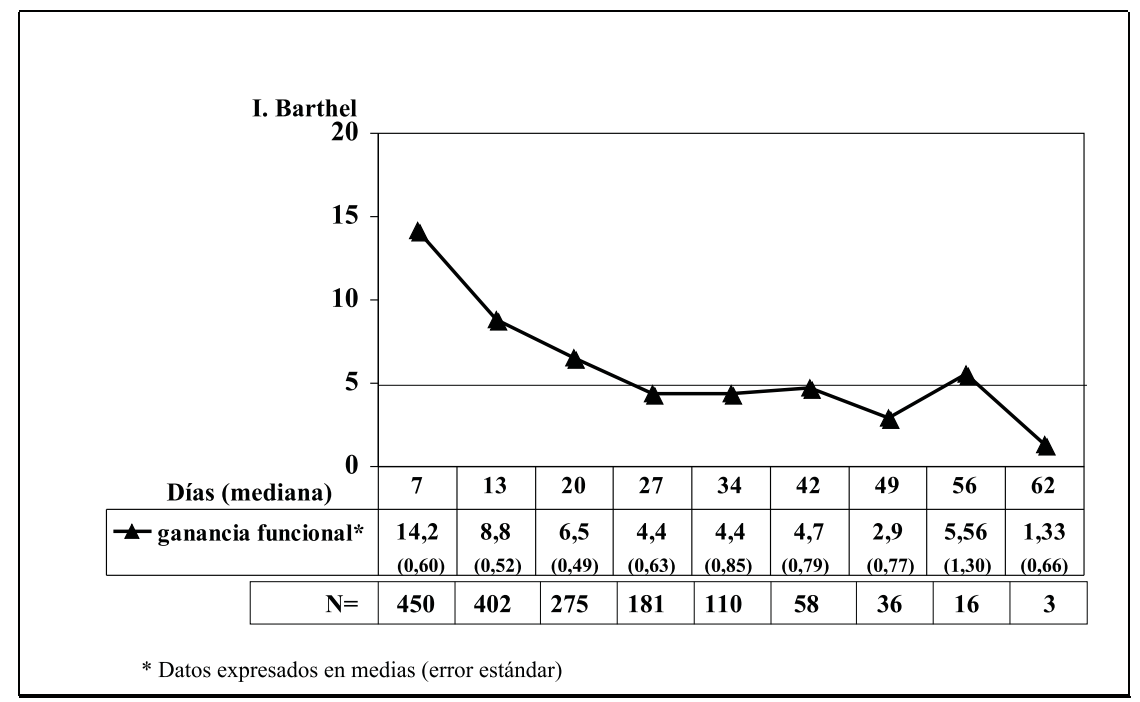

Figura 2

Evolución semanal de la eficiencia de la estancia

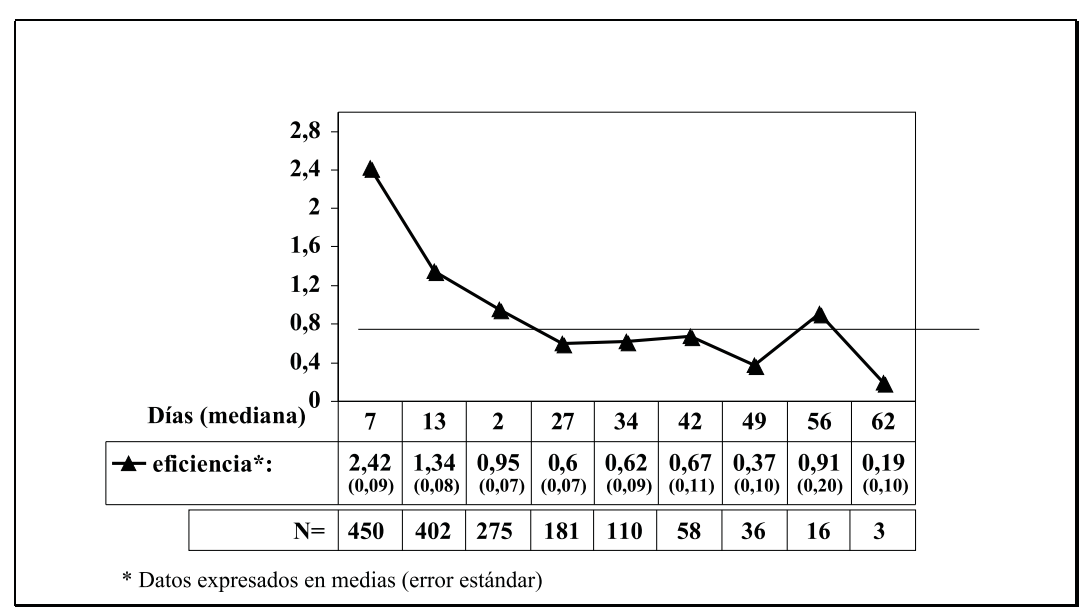

\section{DISCUSION}

El objetivo principal de las Unidades Geriátricas de Media Estancia es la recuperación funcional de los ancianos atendidos en ellas y, de este modo, limitar la incidencia de su institucionalización. Aunque la eficacia en la consecución de estos objetivos ya ha sido comunicada previamente ${ }^{23}$, con frecuencia el proceso de hospitalización está supeditado, además de a las necesidades clínicas del paciente, a la consecución de los 
Figura 3

Evolución semanal de la Ganancia Funcional por grupos de edad

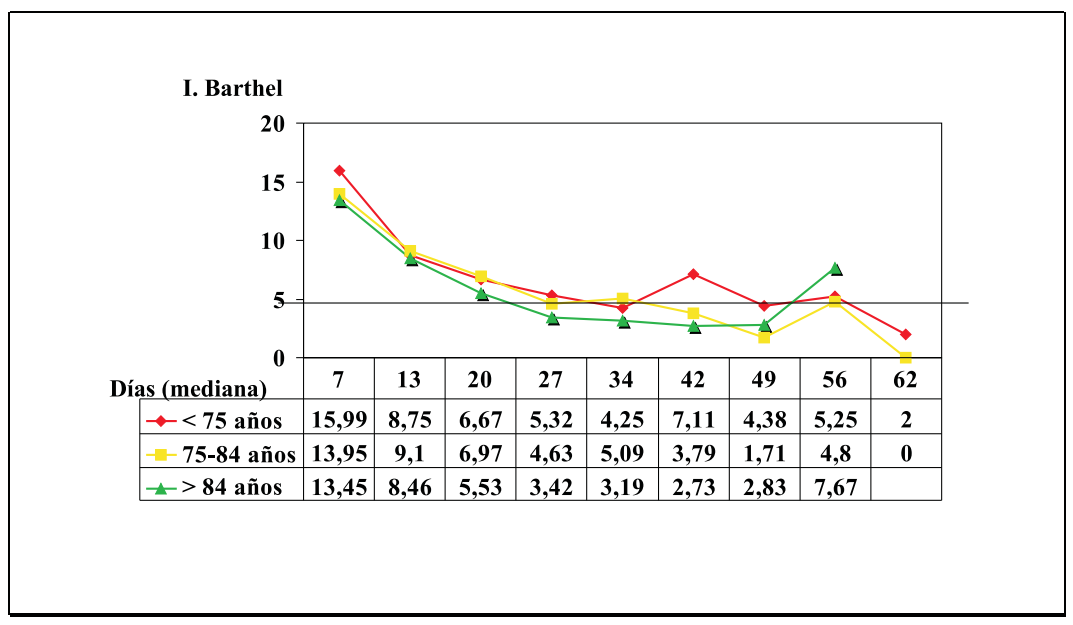

Figura 4

Evolución semanal de la Ganancia Funcional por motivo de ingreso

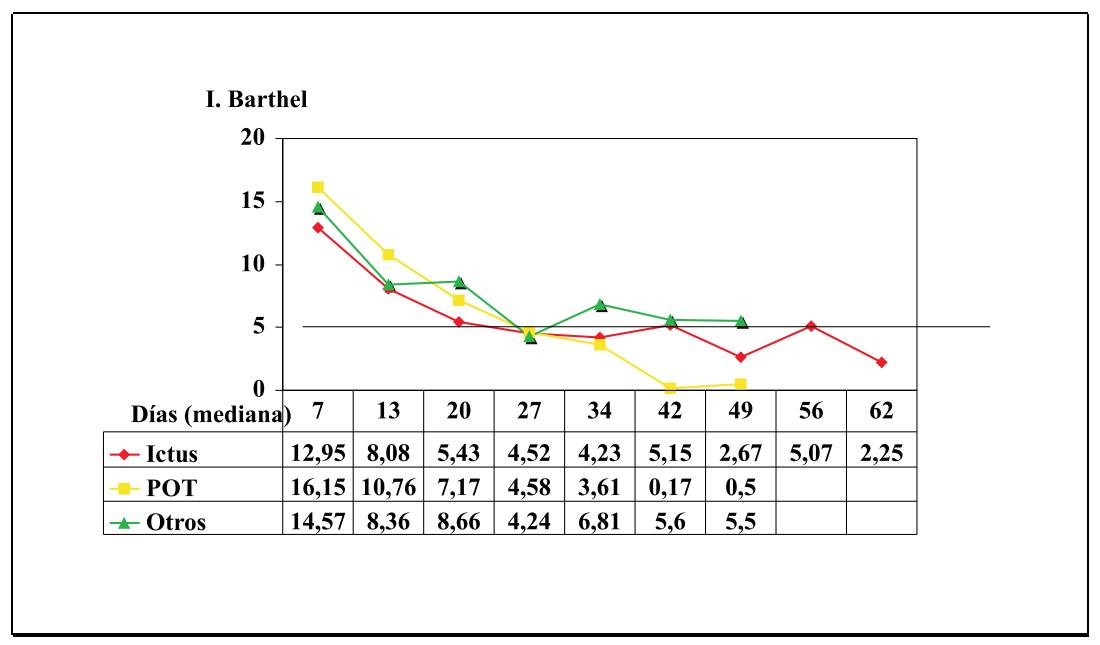

objetivos asistenciales con el uso más eficiente de los recursos existentes ${ }^{5,13}$.

Este estudio pretende acercarse a la evaluación de la estancia hospitalaria en una unidad geriátrica de media estancia a través de la evolución semanal de la ganancia funcional. La asunción de un punto de corte de más de 5 puntos de ganancia en el Indice de Barthel semanal como umbral de eficacia, si bien es arbitrario, viene avalado por recomendaciones derivadas de la práctica clínica 
Tabla 2

Análisis Multivariante de Regresión Lineal de Variables Predictivas de Resultados Asistenciales

\begin{tabular}{|c|c|c|c|}
\hline \multirow{2}{*}{ Variable predictiva } & \multicolumn{2}{|c|}{ Coeficiente de Regresión (p) } \\
\cline { 2 - 4 } & Ganancia Funcional & Estancia & Eficiencia \\
\hline Edad & $-0,11(0,07)$ & $-0,01(0,98)$ & $-0,11(0,07)$ \\
\hline Sexo: varón/mujer & $-0,04(0,44)$ & $-0,03(0,60)$ & $-0,08(0,18)$ \\
\hline Motivo ingreso: ictus/otros & $-0,10(0,08)$ & $0,17(0,001)$ & $-0,17(0,007)$ \\
\hline Días de incapacidad previa al ingreso & $-0,12(0,02)$ & $0,02(0,72)$ & $-0,14(0,02)$ \\
\hline Índice Barthel previo & $0,19(0,002)$ & $0,19(0,001)$ & $0,01(0,89)$ \\
\hline Índice Barthel al ingreso & $-0,45(0,001)$ & $-0,56(0,001)$ & $009(0,18)$ \\
\hline Test de Pfeiffer al ingreso & $-0,21(0,001$ & $-0,19(0,001)$ & $-0,06(0,33)$ \\
\hline Escala socio-familiar Gijón & $0,03(0,58)$ & $0,05(0,32)$ & $-0,07(0,23)$ \\
\hline Índice comorbilidad Charlson & $-0,14(0,01)$ & $0,04(0,44)$ & $-0,07(0,23)$ \\
\hline
\end{tabular}

en pacientes con ictus en unidades de rehabilitación ${ }^{9,10}$. Clínicamente, y pese a no ser una escala continua, esta mejora puede corresponder a la reducción en el grado de dependencia de una de las diez actividades básicas de la vida diaria que evalúa esta escala, y es razonable su aplicación también en la evaluación de la evolución funcional de pacientes ingresados por otras patologías en unidades de media estancia.

En la población estudiada la ganancia funcional es evidente en las tres primeras semanas del ingreso, independientemente del grupo de edad y motivo principal de incapacidad al ingreso. Estos datos reforzarían la idea de que estas unidades requieran una estancia media más prolongada que las unidades de agudos, y que razonablemente puede estar entre 20 y 30 días, tal como se refleja en otras unidades con una rehabilitación intensiva ${ }^{24}$ y como recomendaba el Insalud en 19961. Sin embargo, en nuestro medio las unidades denominadas de media estancia o convalecencia presentan con frecuencia una estancia media superior a 30 días. Este hecho podría estar en relación con una menor dotación de terapeutas, una orientación menos rehabilitadora o la ausencia de una estrategia asistencial interdisciplinaria similar a la presentada en la unidad objeto de este estudio ${ }^{6,25}$.

Un aspecto llamativo es la mejoría funcional que obtienen los pacientes estudiados en su primera semana de ingreso. Este hecho, también comunicado en otros trabajos en nuestro medio ${ }^{26}$, podría estar en relación con el perfil asistencial de estas unidades. La mejoría funcional de la primera semana podría deberse no solo al tratamiento rehabilitador sino también a la orientación de los cuidados en estas unidades, dirigido a limitar las barreras arquitectónicas y asistenciales derivadas de la hospitalización y a no subestimar la capacidad funcional real de los pacientes ancianos atendidos. En otras palabras, ya que el $82 \%$ de los pacientes ingresados procedían de unidades de agudos, este dato podría ilustrar un hecho conocido como es la yatrogenia derivada de la hospitalización en pacientes ancianos motivada por una infravaloración de la situación funcional de estos pacientes así como por una asistencia en unidades de agudos poco favorecedora para integrar los cuidados clínicos con aquellos encaminados a estimular la recuperación funcional del deterioro ocasionado por 
Figura 5

Evolución semanal de la ganancia funcional por unidad de procedencia

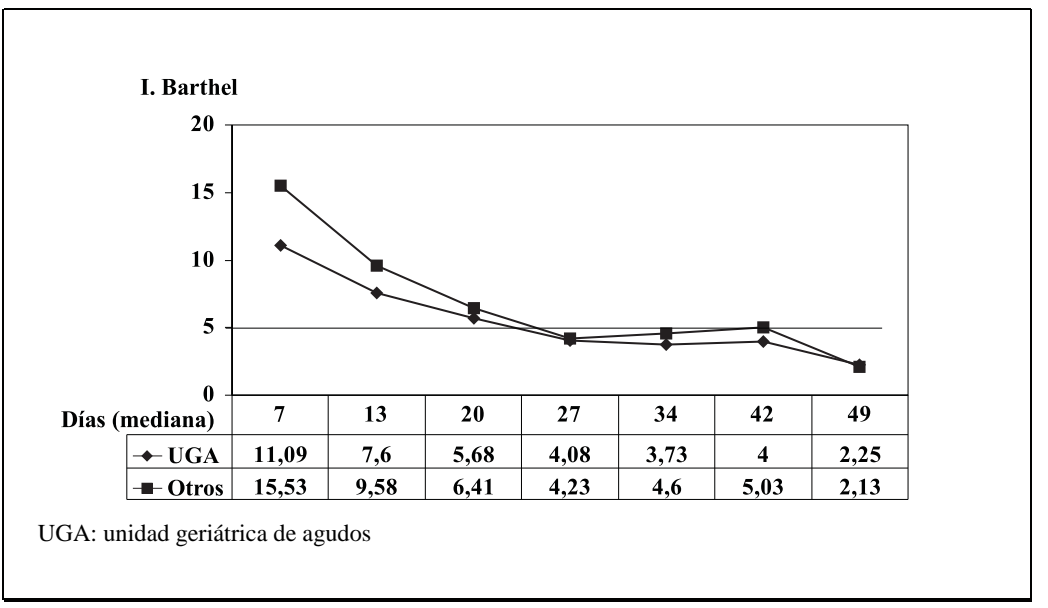

la enfermedad aguda en ancianos frágiles ${ }^{27}$. Este hecho podría justificar la menor ganancia funcional observada en la primera semana de los pacientes derivados la unidad geriátrica de agudos (donde los cuidados centrados en el paciente se dirigen desde el ingreso al control de cuidados clínicos y minimizar su repercusión funcional) comparada con la de los pacientes derivados de otras unidades hospitalarias (figura 5).

A la hora de comparar la estancia hospitalaria entre diferentes unidades es necesario tener en cuenta diversos factores. Así, aquellas unidades con un mayor porcentaje (o dedicadas exclusivamente) a la recuperación funcional de pacientes con secuelas de ictus requieren una mayor estancia, dado que la respuesta de estos pacientes, pasada la fase aguda del evento cerebrovascular, sigue un perfil de recuperación funcional más lento y progresivo $^{2,24}$. Estos pacientes presentaban también una menor ganancia funcional y una menor eficiencia de la ganancia. Sin embargo, conviene resaltar que, aunque pueda resultar paradójico a la luz de sus peores resultados, los pacientes con ictus suelen ser los que más justificación tendrían para ser ingresados en estas unidades geriátricas hospitalarias. Por una parte, es conocido que como consecuencia de la mayor gravedad y repercusión de la patología incapacitante, estos pacientes presentan una menor recuperación funcional que la obtenida por pacientes con fractura de cadera e inmovilidad por otras causas ${ }^{28} \mathrm{y}$, por el contrario, para esta menor recuperación funcional requieren una estancia más prolongada ${ }^{16,24}$. Todo ello se relaciona con una menor eficiencia de la estancia. Sin embargo es esa misma gravedad y complejidad en la recuperación la que justifica su necesidad de tratamiento hospitalario, ya que cuando se comparan los resultados de la rehabilitación de estos pacientes en unidades hospitalarias frente a otras alternativas asistenciales los beneficios evaluados en términos de mejoría funcional e institucionalización a largo plazo son mayores en unidades hospitalarias ${ }^{24}$.

Otros factores podrían influir en la estancia como la situación funcional previa a la causa del deterioro funcional, así como la situación funcional y el estado cognitivo al ingreso. Una mejor situación funcional previa así como una peor situación funcional al 
ingreso se asocian a una mayor estancia, probablemente en relación con la presencia de un mayor porcentaje de pérdida funcional $y$, en consecuencia, un mayor potencial de ganancia funcional que, lógicamente, requiere un mayor tiempo de tratamiento para su recuperación ${ }^{12}$. La menor estancia en relación con la presencia de peor estado cognitivo está en relación con la menor ganancia funcional obtenida por estos pacientes, aspecto ya comunicado previamente $e^{14,16,29}$. Sin embargo, menor ganancia funcional no es sinónimo de ausencia de beneficio funcional en estos pacientes que, aunque más limitado, es clínicamente apreciable ${ }^{15,23}$. Por el contrario, conocer este hecho ayuda a planificar un objetivo asistencial más realista previamente al ingreso, que evite la prolongación innecesaria de la estancia hospitalaria, y que se refleja en la ausencia de asociación entre situación cognitiva y una menor eficiencia de la ganancia funcional ${ }^{16}$.

Frente a otros estudios que relacionaron la edad avanzada con una menor estancia acompañada de una menor ganancia funcional ${ }^{12,30}$, no encontramos relación entre la edad y la estancia, aunque sí una asociación inversa con la ganancia funcional, menor respuesta que podría atribuirse a una mayor fragilidad y menor capacidad de reserva y recuperación asociada con la edad avanzada ${ }^{12,13}$.

De la misma manera, la demora en el ingreso en la unidad desde el inicio de la incapacidad no se asoció con la duración de la estancia, pero sí con una menor ganancia funcional. Este hecho puede estar en relación con que aquellos pacientes con menos complicaciones en la fase aguda inician antes la rehabilitación y tienen una mejor respuesta a la misma ${ }^{12}$. Una segunda lectura es que, independientemente de la causa del retraso del ingreso en la unidad, el aumento de la demora en el inicio de la rehabilitación intensiva repercute negativamente en la capacidad de recuperación de los pacientes, especialmente en los muy ancianos. Igualmente, la relación inversa entre comorbili- dad y ganancia funcional podría ser motivada por la menor presencia de complicaciones en pacientes con menor comorbilidad asociada $^{13,16}$.

Por último, la inclusión del parámetro denominado como eficiencia de la ganancia funcional, que relaciona la ganancia funcional con la estancia hospitalaria, aunque evalúa de una manera grosera el consumo de recursos en este nivel asistencial, es un parámetro sencillo y útil que ayuda a monitorizar la evolución de la actividad de una unidad de media estancia y a comparar sus resultados con los de otras semejantes ${ }^{13}$. Así, valores por encima de 1 reflejan unidades con alta actividad terapéutica y, por el contrario, valores inferiores a 0,5 podrían orientar hacia unidades con una menor actividad terapéutica y mayor frecuencia de cuidados custodiales $^{6}$, lo que indirectamente podría estar indicando un aumento de la estancia hospitalaria inadecuada. Independientemente de estos estándares absolutos, nuestros datos indican que la comparación de la eficiencia entre unidades debería ajustarse por la edad de los pacientes y patología motivo de ingreso.

Algunos estudios han intentado explicar el porcentaje de varianza de la eficiencia de la ganancia funcional en unidades con enfoque rehabilitador. Sin embargo, la mayoría de ellos ${ }^{12,16}$, incluido un estudio preliminar de nuestra población ${ }^{29}$, sólo son capaces de explicar un bajo porcentaje de la varianza de la eficiencia (por debajo del 20\%). Shah et al atribuían este hecho a la influencia de factores sociales, personales y familiares no directamente relacionados con el proceso de rehabilitación, pero que podrían influir tanto en la selección previa de pacientes subsidiarios de ingreso en estas unidades como en la decisión del alta en las mismas ${ }^{12}$. Sin embargo, la inclusión en el modelo de una variable que recogía parte de estas consideraciones (escala socio-familiar de Gijón), no mejoró la varianza de la eficiencia respecto a trabajos previos.

Rev Esp Salud Pública 2004, Vol. 78, N. 3 


\section{BIBLIOGRAFÍA}

1. Insalud. Criterios de ordenación de servicios para la atención sanitaria a las personas mayores. Madrid: Insalud; 1996.

2. Avila R, Vázquez E, Baztán JJ. Unidades de media estancia geriátricas: perspectiva histórica, parámetros de funcionamiento y dilemas actuales. Rev Esp Geriatr Gerontol 2000; 35 (S6): 3-14.

3. Wieland D, Rubenstein LZ, Hedrick SC, Reuben DB, Buchner DM. Inpatient geriatric evaluation and management units (GEMs) in the veterans health system: diamond in the rough?. J Gerontol 1994; 49: M195-200.

4. Young J, Robinson J. Rehabilitation for older people. Br Med J 1998; 316: 1108-9.

5. Landi F, Bernabei R, Russo A, Zuccalà G, Onder G, Carosella L et al. Predictors of rehabilitation outcomes in frail patients treated in a geriatric hospital. J Am Geriatr Soc 2002; 50: 679-84.

6. Baztán JJ, Hornillos M, González-Montalvo JI. Encuesta sobre la estructura y actividad de la unidades geriátricas de media estancia y convalecencia en España. Rev Esp Geriatr Gerontol 2000; 35 (S6): 61-76.

7. Peiró S, Portella E. Identificación del uso inapropiado de la hospitalización: la búsqueda de la eficiencia. Med Clin (Barc) 1994; 103: 65-71.

8. Rentsch D, Luthy C, Perneger TV, Allaz AF. Hospitalization process seen by patients and health care professionals. Soc Sci Med 2003; 57: 571-6.

9. Granger CV, Hamilton BB. Measurement of stroke rehabilitation outcome in the 1980s. Stroke 1990; 21 (suppl II): 46-7.

10. Pinedo S, Miguel de la Villa F. Evolución y pronóstico de la discapacidad en pacientes con hemiplejía. Med Clin (Barc) 2000; 115: 487-92.

11. Heinemann AW, Roth EJ, Cichowski K, Betts HB. Multivariate analysis of improvement and outcome following stroke rehabilitation. Arch Neurol 1987; 44: $1167-72$.

12. Shah S, Vanclay F, Cooper B. Efficiency, effectiveness, and duration of stroke rehabilitation. Stroke 1990; 21: 241-6.

13. Stineman MG, Goin JE, Hamilton BB, Granger CV. Efficiency pattern analysis for medical rehabilitation. Am J Med Quality 1995; 10: 190-8.
14. Valderrama Gama E, Damián J, Guallar E, Rodríguez-Mañas L. Previous disability as a predictor of outcome in a geriatric rehabilitation unit. J Gerontol 1998; 53A: M405-M409.

15. Heruti RJ, Lusky A, Barell V, Orhy A, Adunsky A. Cognitive status at admission: does it affect the rehabilitation outcome of elderly patients with hip fracture?. Arch Phys Med Rehabil 1999; 80: 432-6.

16. Patrick L, Knoefel F, Gaskowski P, Rexroth D. Medical comorbidity and rehabilitation efficiency in geriatric inpatients. J Am Geriatr Soc 2001; 49: 1471-7.

17. Charlson ME, Pompei P, Ales KL, MacKenzie CR. A new method of classifying prognostic comorbidity in longitudinal studies: development and validation. J Chron Dis 1987; 40: 373-83.

18. Shah S, Vanclay F, Cooper B. Improving the sensitivity of the Barthel Index for stroke rehabilitation. J Clin Epidemiol 1989; 42: 703-9.

19. Cid-Ruzafa J, Damián-Moreno J. Valoración de la discapacidad física: el Indice de Barthel. Rev Esp Salud Pública 1997; 71: 127-37.

20. Pfeiffer E. A short portable mental status questionnaire for the assessment of organic brain deficit in elderly patients. J Am Geriatr Soc 1975; 10: 43341.

21. Martínez de la Iglesia J, Dueñas R, Onís MC, Aguado C, Albet C, Luque R. Adaptación y validación al castellano del cuestionario de Pfeiffer (SPMSQ) para detectar la existencia de deterioro cognitivo en personas mayores de 65 años. Med Clin (Barc) 2001; 117: 129-34.

22. Alarcón T, González-Montalvo JI. La escala sociofamiliar de Gijón, instrumento útil en el hospital general. Rev Esp Geriatr Gerontol 1998; 33: 178-9.

23. Baztán JJ, González M, Morales C, Vázquez E, Morón N, Forcano S et al. Variables asociadas a la recuperación funcional y la institucionalización al alta en ancianos ingresados en una unidad geriátrica de media estancia. Rev Clin Esp: en prensa.

24. Kramer AM, Steiner JF, Schlenker RE, Eilertsen TB, Hrincevich CA, Tropea DA et al. Outcomes and costs after hip fracture and stroke: a comparison of rehabilitation setting. JAMA 1997; 277 : 396-404.

25. Salvá A, Martínez F, Llobet S, Vallés E, Miróa M, Llevadot D. Las unidades de media estancia-convalecencia en Cataluña. Rev Esp Geriatr Gerontol 2000; 35 (S6): 31-7. 
26. Cubí D, Roca F, Marco G, López C, Solé M, Arnau A. Functional evolution of activities of daily living (ADL) during the admission to a rehabilitation geriatric unit. Rev Esp Geriatr Gerontol 2003;38 (supl 1): 27.

27. Creditor MC. Hazards of hospitalization of the elderly. Ann Intern Med 1993; 118: 219-23.

28. Johnson MF, Kramer AM, Lin MK, Kowalsky JC, Steiner JF. Outcomes of older persons receiving rehabilitation for medical and surgical conditions compared with hip fracture and stroke. J Am Geriatr Soc 2000; 48: 1389-7.

29. Baztán JJ, Forcano S, González S, Ruipérez I. Rehabilitation outcomes in frail older patients. J Am Geriatr Soc 2003; 51: 281-3.

30. Granger CV, Hamilton BB, Fiedler RC. Discharge outcome after stroke rehabilitation. Stroke 1992; 23: 978-82. 